\title{
On a common generalization of symmetric rings and quasi duo rings
}

\author{
T. Subedi and D. Roy \\ Communicated by A. I. Kashu
}

\begin{abstract}
A BS TR AC T. Let $J(R)$ denote the Jacobson radical of a ring $R$. We call a ring $R$ as $J$-symmetric if for any $a, b, c \in R, a b c=0$ implies bac $\in J(R)$. It turns out that $J$-symmetric rings are a common generalization of left (right) quasi-duo rings and generalized weakly symmetric rings. Various properties of these rings are established and some results on exchange rings and the regularity of left SF-rings are generalized.
\end{abstract}

\section{Introduction}

All rings considered in this paper are associative ring with identity and $R$ denotes a ring. The symbols $J(R), N(R), Z(R), E(R)$ respectively stand for the Jacobson radical, the set of all nilpotent elements, the set of all central elements and the set of all idempotent elements of $R$. We also denote the set $\left\{a \in R: a^{2}=0\right\}$ by $N_{2}(R)$, the ring of $n \times n$ upper triangular matrix over $R$ by $T_{n}(R)$ and the left (right) annihilator of any element $a \in R$ by $l(a)(r(a))$. $R$ is abelian if all its idempotents are central. $R$ is left quasi-duo if every maximal left ideal of $R$ is an ideal. As usual, a reduced ring is a ring without non zero nilpotent elements. $R$ is semiprimitive if $J(R)=0$. $R$ is semicommutative if $l(a)$ is an ideal of $R$ for any $a \in R$. It is well known that $R$ is semicommutative if and only if for any $a \in R, r(a)$ is an ideal of $R$. $R$ is symmetric if for any $a, b, c \in R, a b c=0$ implies $a c b=0 . R$ is reversible if $a b=0$ implies

2010 MSC: 13C99, 16D80, 16U80.

Key words and phrases: symmetric ring, Jacobson radical, $J$-symmetric ring. 
$b a=0$. It is clear that symmetric rings are reversible and reversible rings are semicommutative.

Various generalizations of symmetric rings have been done by many authors over the last several years. $R$ is weak symmetric ([5]) if for any $a, b, c \in R, a b c \in N(R)$ implies $a c b \in N(R) . R$ is central symmetric ([4]) if for any $a, b, c \in R, a b c=0$ implies bac $\in Z(R)$. $R$ is generalized weakly symmetric (GWS) ([11]) if for any $a, b, c \in R, a b c=0$ implies $b a c \in N(R)$. It follows that the class of GWS rings contains the class of weak symmetric rings. Again, it is known that central symmetric rings are GWS ([11]).

\section{Main results}

Definition 1. A ring $R$ is $J$-symmetric if for any $a, b, c \in R, a b c=0$ implies bac $\in J(R)$.

Proposition 1. Following conditions are equivalent for a ring $R$ :

1) $R$ is $J$-symmetric.

2) For any $a, b, c \in R, a b c=0$ implies $a c b \in J(R)$.

Proof. (1) $\Rightarrow(2)$. Let $a, b, c \in R$ such that $a b c=0$ but $a c b \notin J(R)$. Then we get a maximal left ideal $M \subseteq R$ such that $a c b \notin M$ so that $M+R a c b=$ $R$. Therefore $1=x+y a c b$ for some $x \in M, y \in R$. Now (ya)bc=0. As $R$ is $J$-symmetric, byac $\in J(R)$. Thus $(1-x)^{2}=$ yac $($ byac $) b \in J(R) \subseteq M$. Then using $x \in M$ we get $1 \in M$, a contradiction.

$(2) \Rightarrow(1)$. If $a, b, c \in R$ such that $a b c=0$ and $b a c \notin J(R)$, then there exists a maximal left ideal $M \subseteq R$ such that $M+R b a c=R$ which gives $1=x+y b a c$ for some $x \in M, y \in R$. Now $a b(c y)=0$. Then by hypothesis, $a c y b \in J(R)$. Therefore $(1-x)^{2}=y b(a c y b) a c \in M$, whence $1 \in M$, a contradiction. Hence $R$ is $J$-symmetric.

Proposition 2. Let $R$ be a J-symmetric ring and abc $=0$, then for each maximal left ideal $M$ of $R, a \in M$ or $b c \in M$.

Proof. If $a \notin M$, then $M+R a=R$ which implies that $x+y a=1$ for some $x \in M, y \in R$. Then using $a b c=0$ we get $(x-1) b c=0$. As $R$ is $J$ symmetric, $b c(x-1) \in J(R) \subseteq M$ which leads to $b c \in M$.

Corollary 1. Let $R$ be a $J$-symmetric ring, then $N_{2}(R) \subseteq J(R)$.

Corollary 2. Let $R$ be a $J$ symmetric ring, then for any $a, b, c \in R$, $a b c=0$ implies $c a b \in J(R)$.

The proof of the following proposition is trivial. 
Proposition 3. The following conditions are equivalent for a ring $R$ :

1) For any $a, b, c \in R, a b c=0$ implies $c a b \in J(R)$.

2) For any $a, b, c \in R, a b c=0$ implies bca $\in J(R)$.

Proposition 4. If $R$ is a ring such that for any $a, b, c \in R, a b c=0$ implies cba $\in J(R)$, then $R$ is $J$ symmetric.

Proof. Let $a, b, c \in R, a b c=0$ but bac $\notin J(R)$. Then there exists a maximal left ideal $M \subseteq R$ such that $1=x+y b a c$ for some $x \in M$, $y \in R$. Now $a b(c y)=0$. Then by hypothesis we get $c y b a \in J(R)$. Hence $(1-x)^{2}=y b a(c y b a) c \in M$ leading to $1 \in M$, a contradiction. Hence $R$ is $J$-symmetric.

Proposition 5. If $R$ is a left quasi-duo ring and $a b c=0$, then for each maximal left ideal $M$ of $R, a \in M$ or $b \in M$ or $c \in M$.

Proof. Let $M$ be a maximal left ideal of $R$ and $a \notin M$, then $M+R a=R$ which implies that $x+y a=1$ for some $x \in M, y \in R$ leading to $x b c=b c$. As $R$ is left quasi-duo and $x \in M$, we get $b c \in M$. If $b \notin M$, then $M+R b=R$ yielding $u+v b=1$ for some $u \in M, v \in R$, whence $1-v b \in M$ and so $(1-v b) c \in M$. Therefore using $b c \in M$ we obtain $c \in M$.

Proposition 6. A left quasi-duo ring is J-symmetric.

Proof. Let $R$ be a left quasi duo ring and $a b c=0$ and $M$ be a maximal left ideal of $R$. It follows from Proposition 5 that $a \in M$ or $b \in M$ or $c \in M$. As $R$ is left quasi-duo, we get bac $\in M$. Therefore bac $\in J(R)$ which proves that $R$ is $J$-symmetric.

Proposition 7. Central symmetric rings are J-symmetric.

Proof. Let $R$ be a central symmetric ring which is not $J$-symmetric. Then there exists $a, b, c \in R$ such that $a b c=0$ but $b a c \notin J(R)$ so that there exists a maximal left ideal $M \subseteq R$ such that $1=x+y b a c$ for some $x \in M$, $y \in R$. Now for any $r_{1}, r_{2} \in R,(a b)\left(c r_{1}\right) 1=0$ and $\left(r_{2} a\right) b c=0$. Hence $c r_{1} a b, b r_{2} a c \in Z(R)$. Therefore

$$
\begin{aligned}
(1-x)^{4} & =(y b a c)^{4}=y b a c y b a(c y b a c) y b a c=y b a c y b a(b a c c y) y b a c \\
& =y b a c y b a b a c c(y y b a c)=y b a c y b a b a c c(b a c y y) \\
& =y(b(a c y b a b) a c) c b a c y y=y c b a(b(a c y b a b) a c) c y y \\
& =y c b a b a(c(y b) a b) a c c y y=y c b a b(c(y b) a b) a a c c y y \\
& =y c b(a b c) y b a b a a c c y y=0 .
\end{aligned}
$$

This leads to $1 \in M$, a contradiction. Hence $R$ is $J$-symmetric. 
Proposition 8. Generalized weakly symmetric rings are J-symmetric.

Proof. Let $R$ be a generalized weakly symmetric ring and $a b c=0$. If $R$ is not $J$-symmetric, then there exists a maximal left ideal $M$ of $R$ such that $1=x+y b a c$ for some $x \in M, y \in R$. As $R$ is generalized weakly symmetric and $a b c y=0, b a c y \in N(R)$ so that $(b a c y)^{k}=0$ for some positive integer $k$. Therefore

$$
(1-x)^{k+1}=(y b a c)^{k+1}=y(b a c y)^{k} b a c=0 \in M .
$$

This together with $x \in M$ implies that $1 \in M$, a contradiction. Hence $R$ is $J$-symmetric.

Corollary 3. Weak symmetric rings are J-symmetric.

Remark 1. For a field $\mathbb{F}$ and $n>1, R=T_{n}(\mathbb{F})$ is weak symmetric ([5], Proposition 2.3) and hence GWS and $J$-symmetric. As $R$ is not abelian, $R$ is neither central symmetric nor semicommutative. Also, it is worth mentioning here that an abelian ring need not be $J$-symmetric.

Take

$$
R=\left\{\left(\begin{array}{ll}
a & b \\
c & d
\end{array}\right): a, b, c, d \in \mathbb{Z}, a-d \equiv b \equiv c \equiv 0 \quad(\bmod 2)\right\}
$$

Then $E(R)=\{0, I\}$ where $I$ is the identity matrix over $\mathbb{Z}$. Therefore $R$ is abelian. Consider $A=\left(\begin{array}{ll}0 & 2 \\ 0 & 0\end{array}\right)$. Then $A^{2}=0$ but $A \notin J(R)$ as for $K=\left(\begin{array}{ll}2 & 2 \\ 2 & 2\end{array}\right), I-K A$ is not a unit in $R$. Therefore $N_{2}(R) \nsubseteq J(R)$, hence $R$ is not $J$-symmetric.

A ring $R$ is directly finite if for any $a, b \in R, a b=1$ implies $b a=1$.

Proposition 9. Every J-symmetric ring is directly finite.

Proof. Let $a, b \in R$ such that $a b=1$. Take $e=b a$, then $e^{2}=e$. If $c=b(1-e)$, then $c^{2}=0$ so that by Corollary $1, c \in J(R)$ which implies that $a c \in J(R)$ and hence $1-a c=1-a b(1-e)=e$ is invertible which leads to $e=b a=1$.

Recall that a ring $R$ is left min-abel if $(1-e) R e=0$ for any $e \in E(R)$ satisfying $R e$ is a minimal left ideal of $R$.

Lemma 1. For any $e \in E(R), J(e R e)=e J(R) e$ 
Theorem 1. Let $R$ be a J-symmetric ring. Then

(1) If $e \in E(R)$ such that $R e R=R$, then $e=1$.

(2) If $e \in E(R)$ and $M$ be a maximal left ideal of $R$, then either $e \in M$ or $(1-e) \in M$.

(3) $R a+R(a e-1)=R$ for any $a \in R$ and $e \in E(R)$.

(4) $R$ is left min-abel.

(5) For any $e \in E(R)$, eRe is J-symmetric.

Proof. (1) Since $R$ is $J$-symmetric and $R e(1-e)=0, e R(1-e) \subseteq J(R)$. By hypothesis, $R e R=R$ which implies that $R(1-e)=R e R(1-e) \subseteq J(R)$, whence $1-e \in J(R)$ so that $e=1$.

(2) Follows from Proposition 2 as $e(1-e)=0$.

(3) Assume $R a+R(a e-1) \neq R$ for some $a \in R$ and $e \in E(R)$, then there exists a maximal left ideal $M$ of $R$ such that $R a+R(a e-1) \subseteq M$. If $e \in M$, then $a e \in M$, hence $1=-(a e-1)+a e \in M$, a contradiction. If $e \notin M$, then $1-e \in M$ implying $a-a e=a(1-e) \in M$. As $a e-1 \in M$, this leads to $1 \in M$, a contradiction. Hence $R a+R(a e-1)=R$ for each $a \in R$ and $e \in E(R)$.

(4) Let $e \in E(R)$ and $R e$ be a minimal left ideal and $(1-e) R e \neq 0$. Then $R(1-e) R e=R e$. Now $e \in e R e=e R(1-e) R e \subseteq J(R)$ which is a contradiction. Therefore $(1-e) R e=0$ and $R$ is left min-abel.

(5) Let $e \in E(R)$ and eae, ebe, ece $\in e R e$ with $(e a e)(e b e)(e c e)=0$. By hypothesis, $(e b e)(e a e)(e c e) \in J(R)$ and so $e(e b e)(e a e)(e c e) e=$ $($ ebe $)(e a e)(e c e) \in e J(R) e=J(e R e)$ by Lemma 1 .

Converse of (5) of Theorem 1 need not be true. The following example shows this fact.

Example 1. Take $R=M_{2}(\mathbb{F})$, where $\mathbb{F}$ is a field and consider the idempotent $e=\left(\begin{array}{ll}1 & 0 \\ 0 & 0\end{array}\right)$. It is easy to check that $e R e=\left(\begin{array}{ll}\mathbb{F} & 0 \\ 0 & 0\end{array}\right)$ is $J$-symmetric but $R$ is not.

Proposition 10. If $R$ is a J-symmetric ring and idempotents can be lifted modulo $J(R)$, then $R / J(R)$ is abelian.

Proof. Let $\bar{R}=R / J(R)$ and $\bar{a} \in E(\bar{R})$. As idempotents can be lifted modulo $J(R)$, there exists $e \in E(R)$ such that $\bar{e}=\bar{a}$. For any $\bar{x} \in \bar{R}$, write $h=x e-e x e$. Then $h^{2}=0$ and hence by Corollary $1, h \in J(R)$. Therefore $\overline{x e}=\overline{e x e}$, that is $\overline{x a}=\overline{a x a}$. Similarly $\overline{a x}=\overline{a x a}$. Hence $\bar{R}$ is abelian.

Proposition 11. If $R / J(R)$ is symmetric, then $R$ is $J$-symmetric. 
Proof. Let $a, b, c \in R$ such that $a b c=0$. Then $\bar{a} \bar{b} \bar{c}=\overline{0}$. As $R / J(R)$ is symmetric, $\bar{b} \overline{a c}=\overline{0}$ which yields $b a c \in J(R)$. Therefore $R$ is $J$-symmetric.

Proposition 12. Direct product of arbitrary family of $J$-symmetric rings is $J$-symmetric.

Proof. For any arbitrary family of rings $\left\{R_{i}: i \in I\right\}$, we know that $J\left(\prod_{i \in I} R_{i}\right)=\prod_{i \in I}\left(J\left(R_{i}\right)\right)$. Hence the result easily follows.

Corollary 4. A ring $R$ is $J$-symmetric if $e R$ and $(1-e) R$ are $J$-symmetric for any central idempotent $e$.

Example 2. A homomorphic image of a $J$-symmetric ring need not be $J$-symmetric

Consider $\mathbb{Z}_{2}(y)$, the rational functions field of polynomial ring $\mathbb{Z}_{2}[y]$ and $R=\mathbb{Z}_{2}(y)[x]$ be the ring of polynomials in $x$ over $\mathbb{Z}_{2}(y)$ subject to the relation $x y+y x=1$. Now by ([4], Example 2.11), $R$ is central symmetric and therefore $J$-symmetric. Let $L=x^{2} R$, which is a maximal ideal of $R$. Consider $\bar{R}=R / L$. Now $(\bar{x})^{2}=\overline{0}$. So $0 \neq \bar{x} \in N_{2}(\bar{R})$. But $\bar{R}$ being a simple ring, we have $J(\bar{R})=0$. Thus we have $N_{2}(\bar{R}) \nsubseteq J(\bar{R})$, hence $\bar{R}$, a homomorphic image of $R$ is not $J$-symmetric.

The next two propositions gives the condition on an ideal of a ring which forces the ring to be $J$-symmetric.

Proposition 13. Let $I$ be a nil ideal of a ring $R$ such that $R / I$ is $J$ symmetric. Then $R$ is $J$-symmetric.

Proof. Let $a, b, c \in R$ such that $a b c=0$. Then $\overline{a b c}=\overline{0}$ in $R / I$. Since $R / I$ is $J$-symmetric, $\overline{b a c} \in J(R / I)$. Then for any $r \in R$, there exists $t \in R$ such that $1-t(1-r b a c) \in I \subseteq J(R)$ since $I$ is nil. It follows that $(1-r b a c)$ is left invertible and hence $b a c \in J(R)$.

Proposition 14. Let $I$ be an ideal of a $J$-symmetric ring $S$ and let $R$ be a subring of $S$ containing $I$. Then $R / I$ is $J$-symmetric implies $R$ is J-symmertic.

Proof. Let $a, b, c \in R$ such that $a b c=0$ in $R \subseteq S$. Since $S$ is $J$-symmetric, $b a c \in J(S)$. Then for any $r \in R \subseteq S$, there exists $s \in S$ such that $s(1-r b a c)=1$. Now $\overline{a b c}=\overline{0}$ in $R / I$. Since $R / I$ is $J$-symmetric, $\overline{b a c} \in$ $J(R / I)$. Therefore there exists $t \in R$ such that $(1-(1-r b a c) t) \in I$. This yields $s-s(1-r b a c) t \in I$ and so $s-t \in I \subseteq R$. This implies $s \in R$ and hence $(1-r b a c)$ is left invertible in $R$ so that bac $\in J(R)$. 
Proposition 15. Subdirect product of arbitrary family of J-symmetric rings is $J$-symmetric.

Proof. Let $R$ be a subdirect product of a family of $J$-symmetric rings $\left\{R_{i}\right\}_{i \in I}$. Then for each $i \in I$, we have epimorphism $\phi_{i}: R \rightarrow R_{i}$ and hence $\prod_{i \in I} R / \operatorname{Ker}\left(\phi_{i}\right) \simeq \prod_{i \in I} R_{i}$ is $J$-symmetric. The map

$$
\Phi: R \longrightarrow \prod_{i \in I} R / \operatorname{Ker}\left(\phi_{i}\right), \quad \Phi(r)=\left(r+\operatorname{Ker}\left(\phi_{i}\right)\right)_{i \in I}
$$

is a monomorphism. Then $R \cong \operatorname{Im}(\Phi)$. Also $\operatorname{Im}(\Phi) / \Phi\left(\operatorname{Ker}\left(\phi_{i}\right)\right) \simeq$ $R / \operatorname{Ker}\left(\phi_{i}\right)$ is $J$-symmetric. Now $\Phi\left(\operatorname{Ker}\left(\phi_{i}\right)\right) \subseteq \operatorname{Im}(\Phi) \subseteq \prod_{i \in I} R / \operatorname{Ker}\left(\phi_{i}\right)$. Hence by Proposition $14, \operatorname{Im}(\Phi) \cong R$ is $J$-symmetric.

Theorem 2. The following conditions are equivalent for a ring $R$ :

(1) $R$ is $J$-symmetric.

(2) $T_{n}(R)$ is $J$-symmetric for any $n \geqslant 2$.

(3) $R[x] /\left(x^{n}\right)$ is $J$-symmetric for any $n \geqslant 2$.

(4) $S_{n}(R)=\left\{\left(\begin{array}{cccc}a & a_{12} & \ldots & a_{1 n} \\ 0 & a & \ldots & a_{2 n} \\ \vdots & \vdots & \ddots & \vdots \\ 0 & 0 & \ldots & a\end{array}\right): a, a_{i j} \in R, i<j \leqslant n\right\}$ is J-symmetric for any $n \geqslant 2$.

Proof. Let

$$
I=\left\{\left(\begin{array}{ccccc}
0 & a_{12} & a_{13} & \ldots & a_{1 n} \\
0 & 0 & a_{23} & \ldots & a_{2 n} \\
\vdots & \vdots & \vdots & \vdots & \vdots \\
0 & 0 & \ldots & \ldots & 0
\end{array}\right): a_{i j} \in R, i<j \leqslant n\right\}
$$

Then $I$ is a nil ideal of $T_{n}(R)$ as well as $S_{n}(R)$.

$(2) \Rightarrow(1),(3) \Rightarrow(1),(4) \Rightarrow(1)$ are trivial.

$(1) \Rightarrow(2) \cdot T_{n}(R) / I$ is isomorphic to direct product of $n$-copies of $R$. Hence by Proposition 12 and Proposition 13, $T_{n}(R)$ is $J$-symmetric.

$(1) \Rightarrow(3)$. Since $S_{n}(R) / I \simeq R$, it follows that $S_{n}(R)$ is also $J$ symmetric.

$$
\begin{aligned}
& (1) \Rightarrow(4) \cdot R[x] /\left(x^{n}\right) \simeq V_{n}(R) \text { where } \\
& V_{n}(R)=\left\{\left(\begin{array}{ccccc}
a_{0} & a_{1} & a_{2} \ldots & a_{n-1} & a_{n} \\
0 & a_{0} & a_{1} & \ldots & a_{n-1} \\
\vdots & \vdots & \vdots & \vdots & \vdots \\
0 & 0 & 0 & 0 \ldots & a_{0}
\end{array}\right): a_{i} \in R, i=0,1,2, \cdots n\right\} .
\end{aligned}
$$


As $K=I \cap V_{n}(R)$ is a nil ideal of $V_{n}(R)$ and $V_{n}(R) / K \simeq R, V_{n}(R)$ is $J$-symmetric.

If $R$ is $J$-symmetric then $M_{n}(R)$ need not be $J$-symmetric. The following example shows this fact:

Example 3. Let $\mathbb{F}$ be a field and consider $R=M_{2}(\mathbb{F})$. Now $J\left(M_{2}(\mathbb{F})\right)=$ $M_{2}(J(\mathbb{F}))=0$. If $A=\left(\begin{array}{ll}0 & 0 \\ 1 & 0\end{array}\right), B=\left(\begin{array}{ll}0 & 1 \\ 0 & 0\end{array}\right), C=\left(\begin{array}{ll}1 & 1 \\ 0 & 0\end{array}\right)$, then $A B C=0$, but $B A C \neq 0$.

$R$ is (von Neumann) regular if for any $a \in R$, there exists some $b \in R$ such that $a=a b a$. $R$ is strongly regular if for any $a \in R$, there exists some $b \in R$ such that $a=a^{2} b$. It is known that $R$ is strongly regular if and only if $R$ is reduced regular. $R$ is left $S F$-ring if its simple left modules are flat. In 1975, Ramamurthy initiated the study of SF-rings in [10]. It is known that regular rings are left SF-rings. However, till date, it is unknown whether left SF-rings are regular. The regularity of left SF-rings satisfying certain additional conditions have been proved by various authors over the last four deacades (see, [6], [9], [10], [11], [14]). The strong regularity of left (right) quasi-duo left SF-rings, central symmetric left SF rings are proved respectively in [6], [11]. These results are generalized as follows:

Theorem 3. A J-symmetric left SF-ring is strongly regular.

Proof. $R / J(R)$ is a left SF-ring by ([6], Proposition 3.2). Let $b^{2} \in J(R)$ such that $b \notin J(R)$. We claim that $\operatorname{Rr}(b)+J(R) \neq R$. If this is not true, then $1=c+\sum r_{i} t_{i}$, where $c \in J(R), r_{i} \in R, t_{i} \in r(b)$. This yields $b=c b+\sum r_{i} t_{i} b$. Now for each $i,\left(t_{i} b\right)^{2}=t_{i}\left(b t_{i}\right) b=0$ and hence by Corollary $1, t_{i} b \in J(R)$. Therefore $\sum r_{i} t_{i} b \in J(R)$ yielding $b \in J(R)$, a contradiction to $b \notin J(R)$. Therefore $\operatorname{Rr}(b)+J(R) \neq R$ and so there exists a maximal left ideal $M$ of $R$ containing $R r(b)+J(R)$. Since $R$ is a left SF-ring and $b^{2} \in J(R) \subseteq M$, by ([6], Lemma 3.14), there exists some $d \in M$ such that $b^{2}=b^{2} d$, that is $b-b d \in r(b) \subseteq M$, whence $b \in M$. Hence, again there exists some $e \in M$ such that $b=b e$. Then $1-e \in r(b) \subseteq M$, so that $1 \in M$, contradicting $M \neq R$. Therefore $R / J(R)$ is reduced. Hence by ([6], Remark 3.13), $R / J(R)$ is strongly regular. This implies that $R$ is left quasi-duo. Therefore by ([6], Theorem 4.10), $R$ is strongly regular. 
$R$ is clean if every element of $R$ can be written as a sum of an idempotent and a unit. $R$ is exchange if for any $a \in R$, there exists $e \in E(R)$ such that $e \in R a$ and $(1-e) \in R(1-a)$. In [7], Nicholson proved that every clean ring is exchange. Exchange rings need not be clean but under certain additional conditions exchange rings turns out to be clean (see [1], [2], [3], [7], [11], [12]). It is known that left (right) quasi-duo exchange rings are clean ([12]). Also GWS exchange rings are clean $([11])$. These results are extended to $J$-symmetric rings as follows:

Theorem 4. Let $R$ be a J-symmetric exchange ring. Then $R$ is clean.

Proof. Let $x \in R$. By hypothesis, there exists $e \in E(R)$ such that $e \in R x$ and $(1-e) \in R(1-x)$. It is easy to see that $e=y x$ and $1-e=$ $z(1-x)$ for some $y, z \in R$ such that $y=e y$ and $z=(1-e) z$. Therefore $(z e)^{2}=0=[y(1-e)]^{2}$ and so by Corollary 1 , ze, $y(1-e) \in J(R)$. Now $1-z e-y(1-e)=(e-z x+z)-z e-y(1-e)=y x-z x+z-z e-y+y e=$ $(y-z)(x-(1-e))$. As $z e, y(1-e) \in J(R), 1-z e-y(1-e)$ is a unit so that that $x-(1-e)$ is left invertible. Since a $J$-symmetric ring is directly finite, it follows that $x-(1-e)$ is a unit and hence $x$ is clean which implies that $R$ is clean

$R$ has stable range one if for any $a, b \in R$ satisfying $a R+b R=R$, there exists $y \in R$ such that $a+b y$ is a unit. It is known that left (right) quasi-duo exchange rings have stable range one. In [11], Wei proved that GWS exchange rings have stable range one. Observing that a $J$-symmetric ring $R$ satisfies $e R(1-e) \subseteq J(R)$ for any $e \in E(R)$ and using ([8], Theorem $5.4(1)$ ), we get the following theorem which is a generalization of these existing results.

Theorem 5. A J-symmetric exchange ring have stable range one.

\section{References}

[1] E. Akalan, L. Vas, Classes of almost clean rings, Algebr. Represent. Theory 16 (3) (2013), 843-857.

[2] D.D. Anderson, V.P. Camillo, Commutative rings whose elements are a sum of a unit and idempotent, Comm. Algebra 30 (7) (2002), 3327-3336.

[3] V.P. Camillo, H.P. Yu, Exchange rings, units and idempotents, Comm. Algebra 22 (1994), 4737-4749

[4] G. Kafkas, B. Ungor, S. Halicioglu, A. Harmanci, Generalized symmetric rings, Algebra Discrete Math. 12 (2) (2011), 72-84.

[5] L. Ouyang, H. Chen, On weak symmetric rings, Comm. Algebra 38 (2) (2010), 697-713. 
[6] M. B. Rege, On von Neumann regular rings and SF-rings, Math. Japonica 31 (6) (1986), 927-936.

[7] W.K. Nicholson, Lifting idempotents and exchange rings, Trans. Amer. Math. Soc. 229 (1977), 269-278.

[8] Y. Qu, J. Wei, Some notes on nil-semicommutative rings, Turk. J. Math. 38 (2014), 212-224.

[9] T. Subedi, A. M. Buhphang, On SF-rings and regular rings, Kyungpook Math. J. 53 (3) (2013), 397-406.

[10] V. S. Ramamurthy, On the injectivity and flatness of certain cyclic modules, Proc. Amer. Math. Soc. 48 (1975), 21-25.

[11] J.Wei, Generalized weakly symmetric rings, J. Pure Appl. Algebra 218 (2014), 1594-1603.

[12] H.P. Yu, On quasi duo rings, Glasg. Math. J. 37 (1995), 21-31.

[13] H.P. Yu, Stable range one for exchange rings, J. Pure Appl. Algebra 98 (1995), 105-109.

[14] H. Zhou, Left SF-rings and regular rings, Comm. Algebra 35 (2007), 3842-3850.

\section{CONTACT INFORMATION}

Tikaram Subedi, Department of Mathematics, National Institute Debraj Roy of Technology Meghalaya, India

$$
\begin{gathered}
E \text {-Mail(s): tsubedi2010@gmail.com, } \\
\text { debraj.hcu@gmail.com }
\end{gathered}
$$

Received by the editors: 24.06.2017. 